\title{
Design of a Climbing Robot for Inspecting Aircraft Wings and Fuselage
}

\author{
Jianzhong Shang, Tariq P.Sattar, Shuwo Chen, Bryan Bridge \\ London South Bank University - Department of Electrical, Computer and Communications Engineering \\ 103 Borough Road, London, UK \\ email: shangj@1sbu.ac.uk, sattartp@1sbu.ac.uk
}

\begin{abstract}
This paper focuses on the design of a wall climbing robot. The robot carries a Cartesian scanning arm and a payload of various non-destructive testing (NDT) sensors while walking on the topside and downside of wings and on varying surface curvatures presented by the fuselage of different types of aircraft. The robot uses pneumatic cylinders to actuate the robots motion in $\mathrm{X}$ and $\mathrm{Y}$ directions. It uses suction cups to adhere the robot to the surface. The main achievement of this robot is the capability to cope with varying surface curvature when climbing around the aircraft while carrying a payload of up to $18 \mathrm{~kg}$. The robot achieves this capability with sufficient flexibility in its structure, feet and suctions cups to cope with varying surface curvature while remaining rigid once the robot feet adhere to the surface. Robot rigidity ensures a stable climbing motion and the vibration free deployment of the NDT sensors.
\end{abstract}

Keywords: Climbing Robots, Surface Adaptation, Aircraft Inspection

\section{INTRODUCTION}

Regular and periodic Non-Destructive Evaluation (NDE) is mandatory for civil airlines throughout the world. Most inspection is currently carried out manually. Manual inspection of large surfaces is tedious and leads to operator fatigue and hence mistakes in defect detection.

Increasingly, airlines require a hard copy of inspection results to eliminate operator subjectivity. Furthermore, the requirement for $100 \%$ inspection of vital structural features is becoming more commonplace. Mobile robotics offers a means to provide access to large surfaces and carry out accurate deployment of NDT sensors thereby improving defect data acquisition and eliminating operator tedium and fatigue.

The objective of this project $[1,2,3]$ was to develop a robotic inspection system, which will walk over large areas of an aircraft structure, scanning rows of rivets and carrying out data collection and interpretation to identify structural defects, loose rivets and cracks in the aircraft skin.

There are very few methods that wall climbing robots can use to hold a robot onto wall surfaces. These are vacuum suction cups, permanent or electro-magnets, propeller thrust forces, vacuum created with vortex fans, and to a lesser extent the use of special adhesive materials. Since aircraft fuselage and wings are constructed from non-ferrous materials, using magnets to adhere the robot to the aircraft surface cannot be used. From these methods, vacuum suction cups provide the best means to control and generate sufficient adhesion force to hold the robot and large payloads.

The general requirements for the robot include:
(1) the ability to climb on the top-side and down-side of aircraft wings and all the surface around the fuselage, moving in any direction

(2) the capability to negotiate varying surface curvatures as the robot travels to different parts of aircraft, with the radius of curvature being as small as 1.5 meters on the fuselage to nearly infinity on some parts of wings

(3) the capability of carrying a payload of up to $18 \mathrm{~kg}$. The payload is composed of NDE equipment, and either a Cartesian scanning arm mounted with NDE sensors or a thermographic camera and heating gun. The Cartesian scanner and the thermograhic system are mounted on separate platforms so that they can be quickly replaced for different tasks with a quick change mechanism

(4) light weight. For the convenience of the end user, the robot should be carried and lifted to be placed on the working surface by at most two operators. Therefore, the robot should be compact and light.

The characteristics of the robot are listed in Table 1.

Table 1 Characteristics of the climbing vehicle

\begin{tabular}{|l|l|}
\hline Mass of climbing vehicle & $20 \mathrm{~kg}$ \\
\hline Outer Dimensions & $518 \times 518 \times 180 \mathrm{~mm}$ \\
\hline $\begin{array}{l}\text { Payload including } \\
\text { umbilical mass }\end{array}$ & $18 \mathrm{~kg}$ \\
\hline Payload Platform size & $300 \times 300 \mathrm{~mm}$ \\
\hline Speed, straight-line motion & $600 \mathrm{~mm} / \mathrm{min}$ \\
\hline Motion & Step size $50 \mathrm{~mm}$, Rot $\pm 5^{\circ}$ \\
\hline Electrical Supply & 24 VDC \\
\hline Air supply & $\begin{array}{l}\text { Supply max }=10 \text { bars, } \\
\text { Operation } 7 \text { bars } \\
\text { Volume }=5801 / \text { min }\end{array}$ \\
\hline $\begin{array}{l}\text { Communication between } \\
\text { vehicle/console }\end{array}$ & RS 485 \\
\hline $\begin{array}{l}\text { On-board Vehicle Control } \\
\text { system }\end{array}$ & $\begin{array}{l}\text { Digital I/O, One Servo } \\
\text { system and } \\
\text { micro-controller }\end{array}$ \\
\hline Umbilical & $\begin{array}{l}\text { One } 12 \mathrm{~mm} \text { air pipe, One } \\
\text { RS } 485 \text { Communication } \\
\text { cable, One power supply } \\
\text { cable }\end{array}$ \\
\hline
\end{tabular}

\section{RoBOt DESIGN}

\section{A. General structure of the robot}

Three designs of climbing robot, as shown in Figure 1, were evaluated. Each design has its advantages and disadvantages. The design in (c) was finalized and prototyped. In this structure, there are two pairs of rodless pneumatic cylinders that drive the 
robot motion in two directions. One pair of cylinders, parallel to each other, move together to drive the robot motion along $\mathrm{X}$ axis, called the $\mathrm{X}$-cylinders. The other pair drives the robot motion along $\mathrm{Y}$ axis, called the $\mathrm{Y}$-cylinders. The two $\mathrm{X}$ cylinders are mounted on two sides of a platform called X-platform, while the two Y cylinders are connected by the Y-platform. The two platforms are normal to each other when they are in the neutral position. Between the two platforms, there is a rotating mechanism driven by a motor. This mechanism adjusts the angle between the two platforms by \pm 5 degrees to correct for off-course deviations. At two ends of each cylinder, there are two "leg" cylinders to drive the feet up and down. The feet that are mounted on the ends of the X cylinders are located at the four corners, they are also called the "outer group". The feet that are mounted on the ends of the Y cylinders, also called "inner group", are extended to the edges of the robot to increase the capability of anti-overturning moment. Each foot is constructed of a plate with four small suction cups.

This robot climbs in a stepping gait with two groups of foot working alternatively. When outer group adhere the robot to the surface, the $\mathrm{X}$ or $\mathrm{Y}$ cylinders drive the inner group moving one cylinder stoke distance in $\mathrm{X}$ or $\mathrm{Y}$ direction respectively, then change to the inner group to adhere the robot and drive the outer group moving. The two groups of foot work alternatively in such a way that the robot moves step by step.

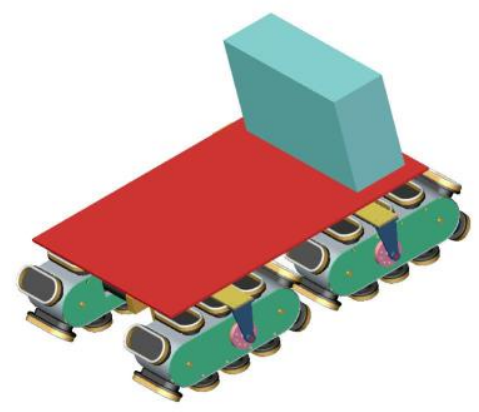

(a)

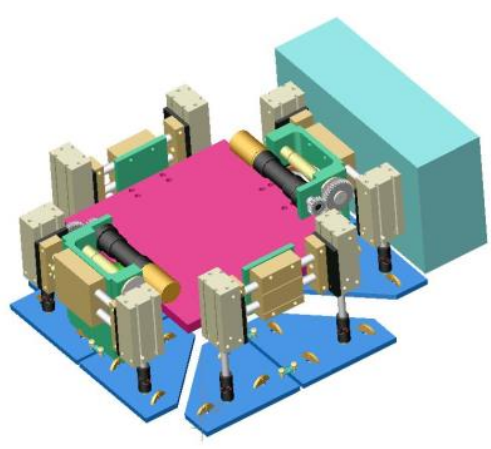

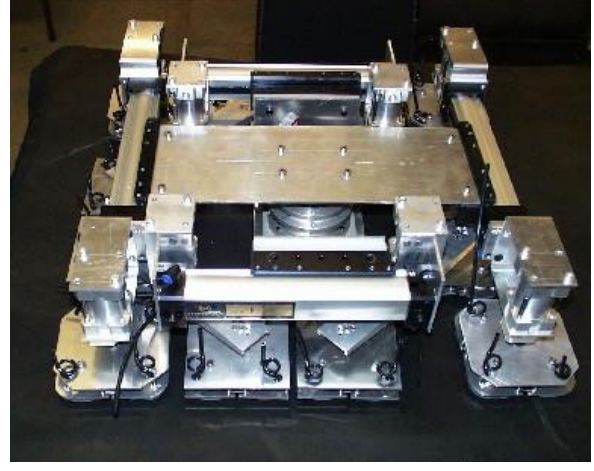

(c)

Fig. 1. Overall structure of the robot designs

\section{B. Stability analysis of the multi support point structure}

From the basic geometrical axiom that "through three noncolinear points, there is exactly one plane", we define, from a view of mechanics, this plane as intrinsically stable. In terms of the robot design, the distinct stable structure is using three feet to support the robot. However, there are some disadvantages with three feet structure. (1) Three feet cover less area, therefore generate less holding force. The holding force generated by a suction cup is determined by the area of the cup and the vacuum level. With a given vacuum level, the bigger the area that the suction cups cover, the more the force available to hold the robot. (2) Less capability to negotiate curvatures. In order to achieve a bigger holding force, larger suction cups are preferred. The cup skirts are made from soft material that can be compressed to seal the cup on a curvature. However, larger cups tend to cause vacuum leakage as illustrated in Figure 2 (a) and (b). When the cup contacts to the surface, there is a gap $h$ between the edge of the cup and the surface. When force $f$ is applied, the gap reduces to $h-d$, where $d$ denotes the deformation of the cup under the force $f$. If $h>d$, the gap cannot be filled up and thus causes vacuum leakage. With small cup as shown in (c)-(f), the gap $h$ between the edge of the cup and the surface is small, when the same force $f$ is applied, the deformation $d$ is more than the gap $h$, so the gap is filled up. (3) No tolerance for vacuum failure. In a three suction cup structure, if one of them loses vacuum, the three support point condition is no longer satisfied, the other two cups will experience a peel force that is likely to peel off the robot. Unfortunately, to entirely prevent suction cups that work on imperfect surfaces from losing vacuum is difficult. The good solution is to divide one big cup into small cups, should some of them fail to function, the others are still able to hold the robot. 

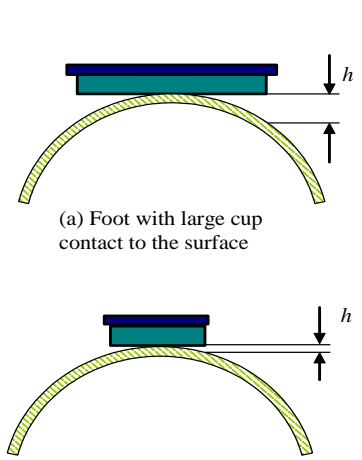

(c) Foot with small cup

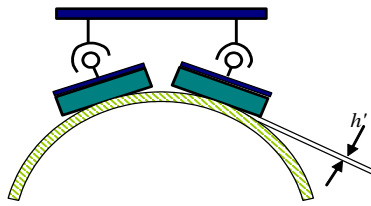

(e) Foot with small cups

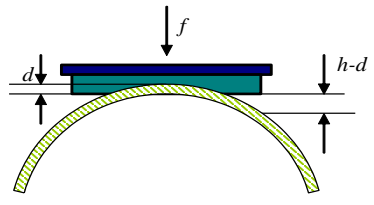

(b) Foot with large cup under force $f$

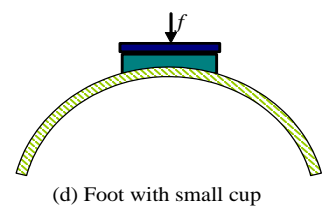
under force $f$

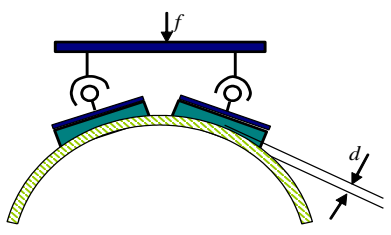

(f) Foot with small cups under force $f$

Fig. 2.

Based on the discussion above, a multi-point support structure is designed, which employs 16 cups for the outer group and 32 cups for the inner group. That is 4 feet in outer group and 8 feet in inner group.

A stable foot structure is designed as shown in Figure 3. There are four cups on each foot, two of them have individual joints and the other two share one joint. This design can be explained in a quadtree structure, as shown in Figure 3(a). A, B, $\mathrm{C}$ and $\mathrm{D}$ are four suction cups on one foot, which might cause instability. If the $\mathrm{B}$ and $\mathrm{D}$ are connected and brought to $\mathrm{E}$, the structure becomes three non-collinear support point (A, C and E) stable structure.

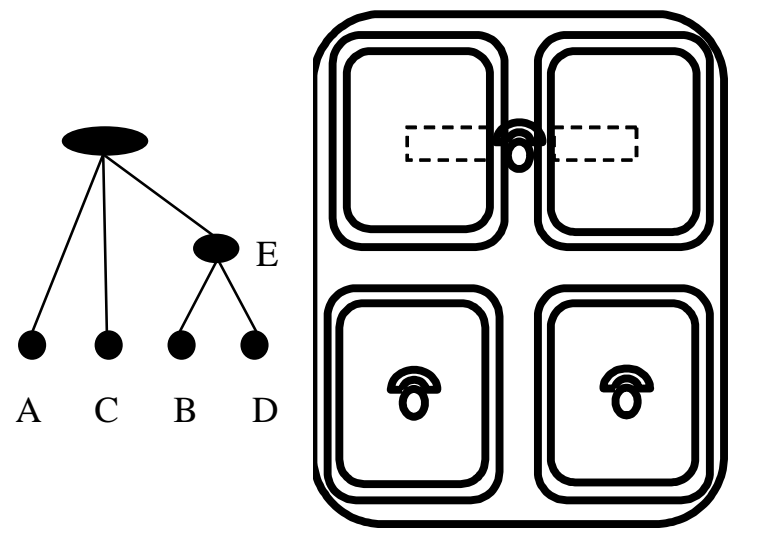

(a)

(b)

Fig. 3. A stable foot structure

The principle of intrinsic stability of four point structure discussed above can be extended to the multi support point structure. Thus the general design guide for the intrinsically stable climbing vehicle with multi suction cups can be established.

An $\mathrm{N}(\mathrm{N}>3)$ support point structure can be equivalently transformed to a structure of three non-collinear support points by dividing $\mathrm{N}$ into three groups. If any one or more groups have more than three points, then they can be further divided, and finally the structure is transformed to three support point structure. The transformed structure fulfils the intrinsic stability possessed by a three non-collinear support point structure. Figure 4 illustrates how the outer platform with 16 support points fulfill the intrinsic stable principle.

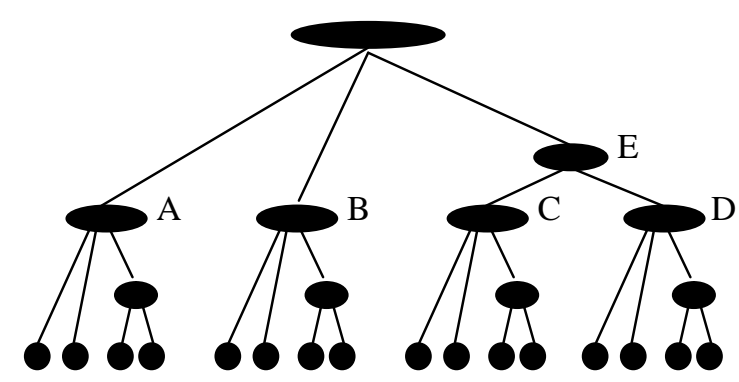

Fig. 4. Stable multi support point structure

\section{Flexibility and stability of the robot to adapt to surfaces with unforeseen curvatures}

The design has been proven to fulfill the three non-collinear support point intrinsic stable principle. However, in order to make the robot flexible to adapt to varying curvature, each cup is held by an universal joint, each foot is also held by an universal joint. In the internal group, one pair of foot is connected by a metal bar and the bar is connected to the 'leg' cylinder by an universal joint too. This flexible design makes the robot easily adapt to curvatures, but the stability of this structure needs to be proved. We take the structure in Figure 5 (c) as an example. The structure illustrates a platform, a cylinder pair for the movement, four "leg" actuators and four feet. Each foot can be assumed as a rigid element at this stage. One cylinder with two "legs" and "feet" is mounted on the platform rigidly, whilst another cylinder with two "legs" and "feet" is connected to the platform by a cylindrical joint. If the number of freedom is 0 , then we say the structure is stable. In this structure, the number of the degrees of freedom, $F$, is given by,

$$
F=6 N-5 P_{5}-4 P_{4}-3 P_{3}-2 P_{2}-1 P_{1}
$$

Where

$\mathrm{N}$ - number of moving parts

$\mathrm{P}_{\mathrm{n}}$ - number of joints introduced to restrain $\mathrm{n}$ degrees of freedom, $\mathrm{n}=1,2, \ldots 5$.

We say each "leg" is an element, as shown in Figure 5 (a), the foot is connected by a universal joint. Once the foot is adhered to the surface, the lower half is fixed and only the top half is moveable. The three degrees of freedom that the leg translates along the $\mathrm{X}, \mathrm{Y}$ and $\mathrm{Z}$ axis are restrained, but the leg still has the degree of freedom rotating about axes $\mathrm{X}, \mathrm{Y}$ and $\mathrm{Z}$. In this case,

$$
F=6 \times 1-1 \times 3=3
$$

If two legs are connected together, as shown in Figure 5 (b), there is still only one moving part and joint 1 restrains three degrees of freedom as in the single element, two degrees of freedom, rotating about axes $\mathrm{X}$ and $\mathrm{Z}$, are restrained because of the second joint applied. From the equation1, there is 


$$
F=6 \times 1-1 \times 3-1 \times 2=1
$$

The degree of freedom is one, which is rotating about axis $\mathrm{Y}$.
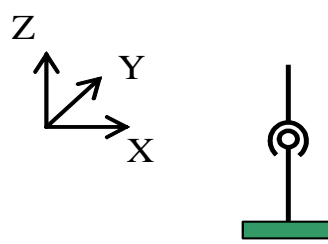

(a)

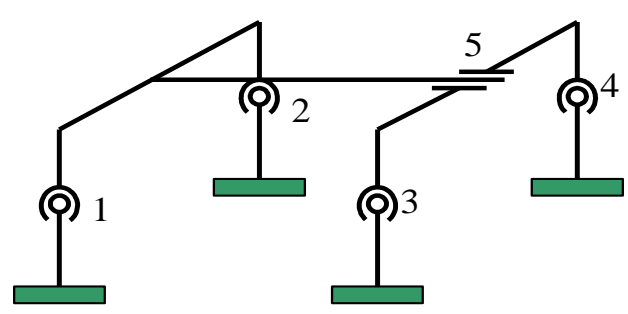

(c)

Fig.5. Structure of four legs

As the structure in (c), once the feet adhere to the surface, the number of moving parts is 2 , joints 3 and 4 are the same as 1 and 2 , the joint 5 restrains the degree of freedom rotating about axis $\mathrm{Y}$ for both of the moving parts, so

$$
F=2 \times 6-2 \times 3-2 \times 2-1 \times 2=0
$$

We can conclude that this structure is stable. The same analysis can be extended to the foot structure, and the outer assembly structure.

\section{Other techniques used in the robot design}

\section{1) Pressure regulation}

In the case of changing legs, one group of foot is placed down while the other group adheres to the surface. The force that places the feet down intends to lift the other group up, and if this force is greater than the suction force that is currently holding the robot, the feet will be pulled off. To prevent this happening, the pressure that places the feet down is regulated. The pressure is initially set to 1 bar, then vacuum ejectors are enabled and the vacuum status is checked. If the vacuum is generated reliably, release the other group and apply the full pressure to lift the robot body up for next movement. Otherwise, increase the pressure and check the vacuum status again. If the pressure reaches a threshold that possibly pull the other group off before the vacuum generated reliably, the robot is stopped to wait for further instruction.

\section{2) Vacuum sensing}

There is one vacuum sensor on each foot, and the vacuum is sensed when a group of feet is placed on the surface and the vacuum enabled. In a good condition where there is little vacuum leakage, $90 \%$ vacuum is achievable. However, in real condition, vacuum leakage always happens. The vacuum sensor is set to $60 \%$, so that even if there is vacuum leakage, as long as the foot is getting more than $60 \%$ vacuum, the robot can continue the movement.

\section{3) Flow regulation}

In order to reduce the size of the umbilical, the cylinders and vacuum ejectors consume the same compressed air supply. Any sudden drop of the air pressure could cause loss of vacuum. For safety reason, the air supply priority is given to the vacuum ejectors. In other words, the air flow to drive the pneumatic cylinders is regulated so that the air pressure does not drop down significantly while the cylinders are moving.

\section{E. Control system of the robot}

In this control system, the robot carries on-board control modules that control the robot movement and get feedback from the sensors. The host computer is on the ground. Communication between the host computer and the on-board controllers is via RS485 serial cable. High level motion instructions are given from the host computer. The on-board controllers translate and execute it. There are one servo module and two IO modules to control the robot movement, as shown in Figure 6. The servo module controls a DC servo motor to correct the off-course deviations. The digital IO ports on the IO modules are direction programmable for controlling the pneumatic valves and vacuum ejectors and getting signals from the sensors. The analog signal for the pressure regulation is given from the analog output embedded in the IO modules.

The modules are compact and light weight so that they can be carried on the robot without putting on too much payload. The single serial communication cable also minimizes the robot umbilical. The control system can easily be reconfigured by using different combination of modules, such as servo modules, stepper modules and IO modules, in order to adapt to other tasks.

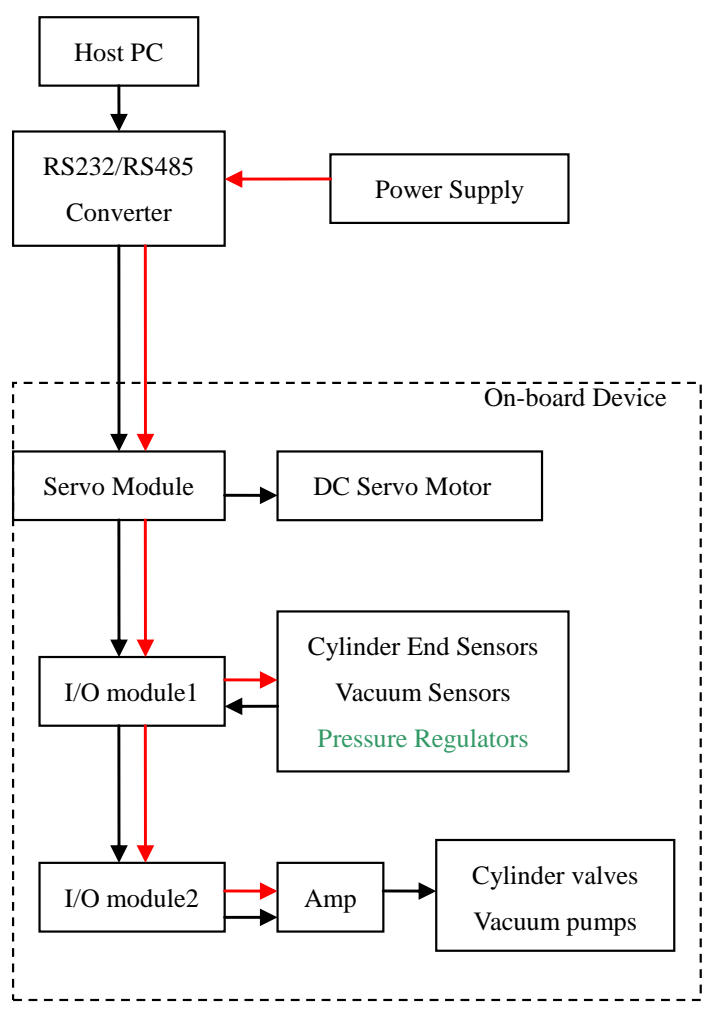

Fig. 6. Control diagram 


\section{NDE METHODS AND SENSORS}

A number of NDE methods and equipments are developed or used in this project. They are not designed to work simutaniously, but there is a quick change mechnism that enables quick replacement of the equipments for different tasks.

\section{A. Phased Array Sensor}

The foremost advantage of phased array system is that the ultrasonic beam is electronically steered instead of raster scan in conventional ultrasonic inspection, so that the inspection is more efficient. A phased array scan image with defect next to rivets is shown in Figure 7.

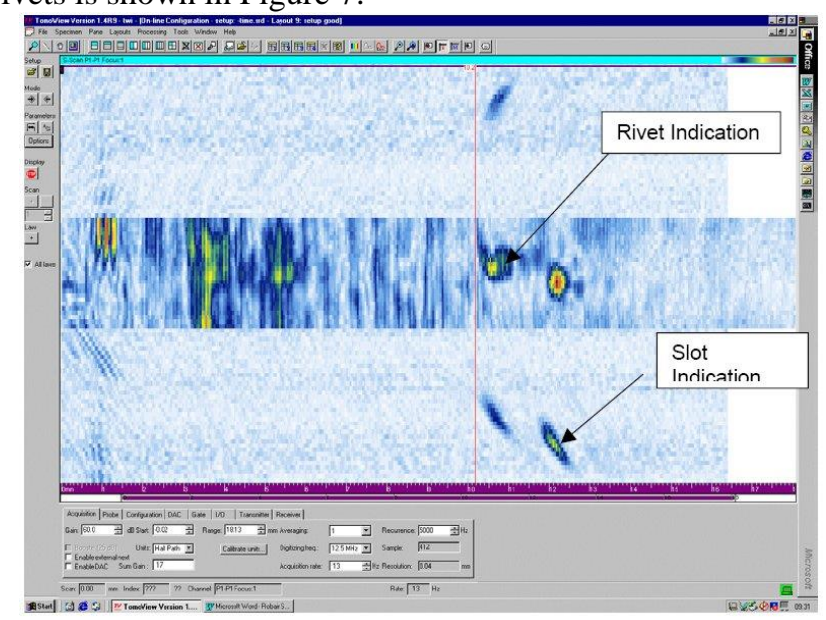

Fig. 7. Phased array signal from slot next to fastener

\section{B. Eddy Current System}

The eddy current system is required to do two tasks: firstly standard eddy current inspection for surface cracks and sub-surface corrosion and secondly to locate the fasteners to enable phased array inspection to be carried out. Eddy current probes scan the surface of the fuselage/aircraft wing etc to locate and determine whether or not any of the rivets have any defects. An eddy current test image is shown in Figure 8, where the fasteners are displayed clearly and the defect is indicated. The position of the fasteners is located with an accuracy of $+/-1 \mathrm{~mm}$.

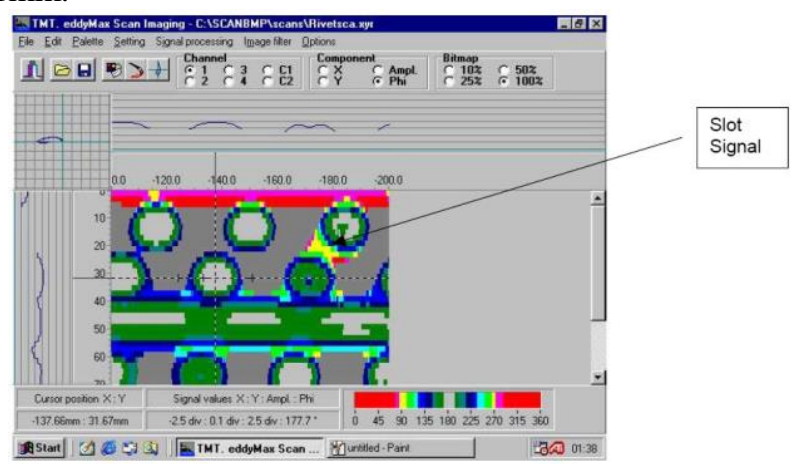

Fig. 8. Eddy current test showing fasteners and defect indication

\section{Thermographic System}

Thermographic techniques to detect loose rivets and for other applications are potentially a very rapid process. For the technique to work, it is essential that the heat source is removed completely whilst the thermal imager scans the decay period. Our system uses a hot air source and directs the air through a nozzle for a given time and then mechanically rotates it away from the surface. Figure 9 shows the difference that the temperature changes against time when loose rivet and tight rivet are heated and cooling down.

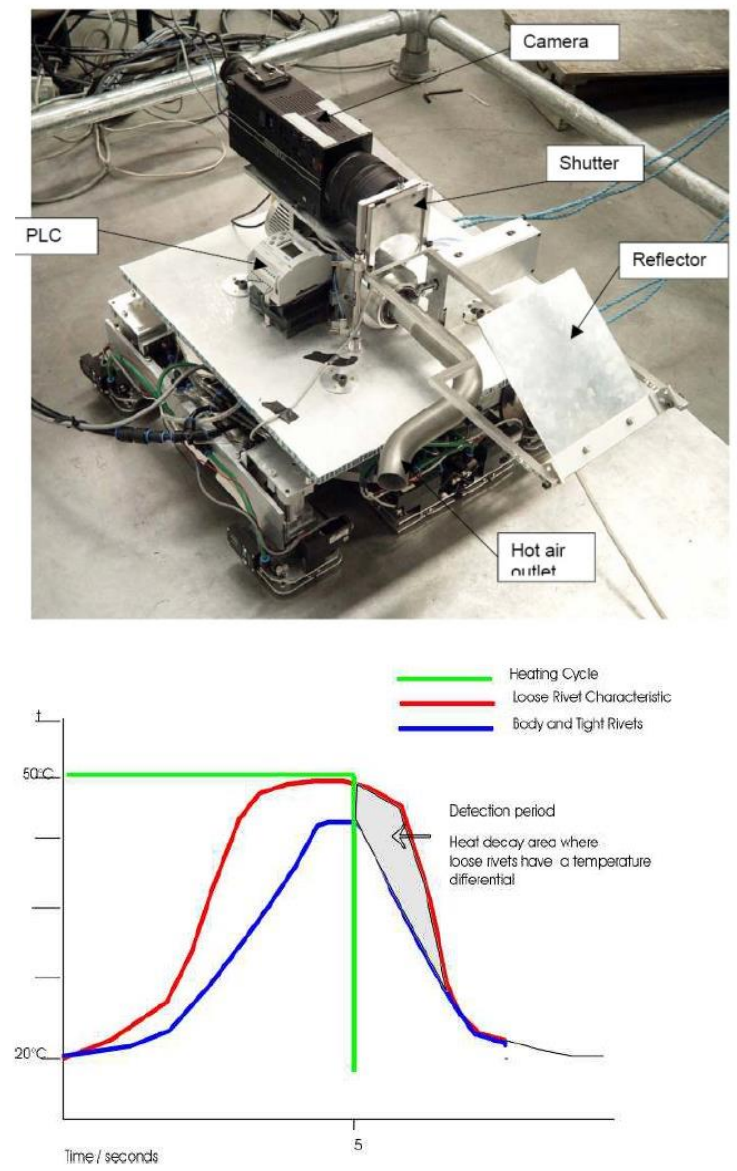

Fig. 9. Thermographic test

\section{Solid Coupled Wheel Probe}

Probes made of a thick hydrophilic polymer tyre that couples to the test surface and a $10 \mathrm{~mm}$ diameter immersion probe that is held within the fluid filled wheel are used to identify impact damage on composite wings and to measure aluminium skin thickness. The device operates in pulse echo mode. The use of hydrophilic material reduces the amount of water required to achieve consistent coupling and being a wheel of soft material it does not damage the surface. This technology is specially useful for robotic inspection as it eliminates the use of couplant and performs 'clean' inspection. 


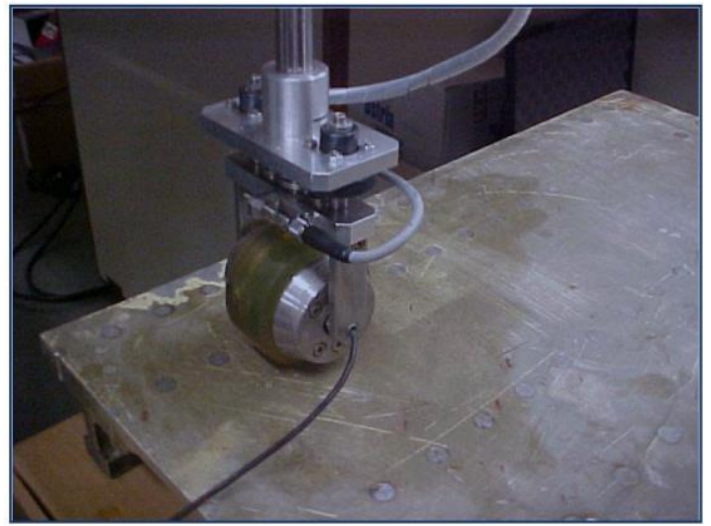

Fig. 10. Wheel probe under test

\section{RoBOt Tests}

The robot was firstly tested in our lab on a curved aluminium plate carrying a dummy payload of $18 \mathrm{~kg}$. It was tested climbing horizontally, vertically and also on the downside of the plate. Then, the robot was tested on a real fuselage section of a DC10 aircraft with the scanning arm and other NDT equipments, as seen in Figure 11. It was able to carry the specified payload and climb on the surface of the fuselage, and adhere itself on the surface rigidly for the inspections.

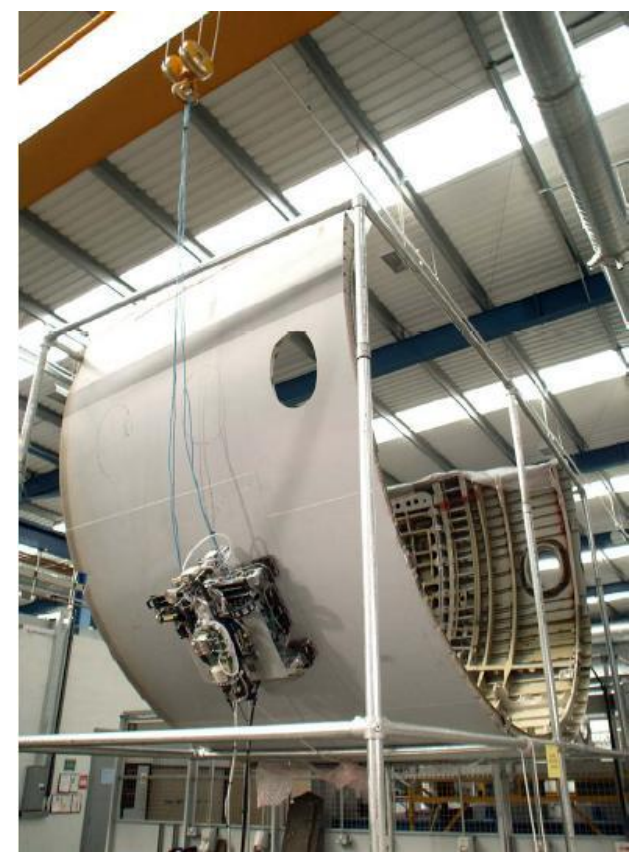

Fig. 11. Robot climbing on a D10 aircraft fuselage section

\section{CONCLUSION}

The design of a climbing robot that has flexible suction cups to ensure the correct creation of vacuum, and a flexible structure to adapt to varying surface curvatures, is able to maintain its rigidity when the vacuum suction cups are active. The rigidity is sufficient to correctly support the NDT inspection carried out.

\section{ACKNOWLEDGMENTS}

The ROBAIR project is funded by the European Community, Contract G4ST-CT-2000-50028 ROBAIRPROJECT $\mathrm{N}^{\circ}$ : CRAF-1999-70040, ACRONYM: ROBAIR. The project partners are Sonatest Plc., NDT Consultants Ltd., Zenon SA Robotics \& Informatics, Kontroll Technik GmbH, Horton Levi Ltd, Technical University of Sofia, and Ideasis. The project is coordinated and managed by TWI Ltd.

\section{REFERENCES}

[1] European Community project ROBAIR, Development of a Robotic System for the Inspection of Aircraft Wings and Fuselage, $5^{\text {th }}$ Framework Programme, project No. CRAF-1999-70040, 1998-2002.

[2] Tariq P. Sattar, Shuwo Chen, Bryan Bridge, Jianzhong Shang ROBAIR: Mobile Robotic System to Inspect Aircraft Wings and Fuselage, Proc. 19th International Conference on CAD/CAM, Robotics and Factories of the Future, Vol. 2, pp 795-802.

[3] Bridge, Bryan and Sattar, Tariq P. Total Integrated Robotic Structural Inspection for Enhanced Aircraft Life and Safety, Proc. 17th International Conference on CAD/CAM, Robotics and Factories of the Future, 2001, Vol. 1, pp. 444-453, ISBN Number: 1-86840-432-3.

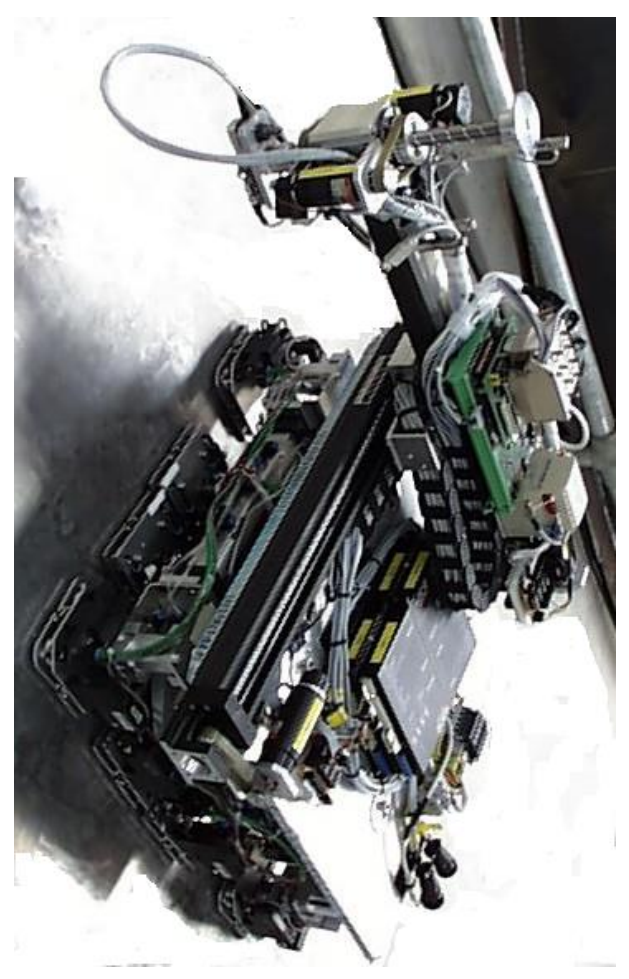



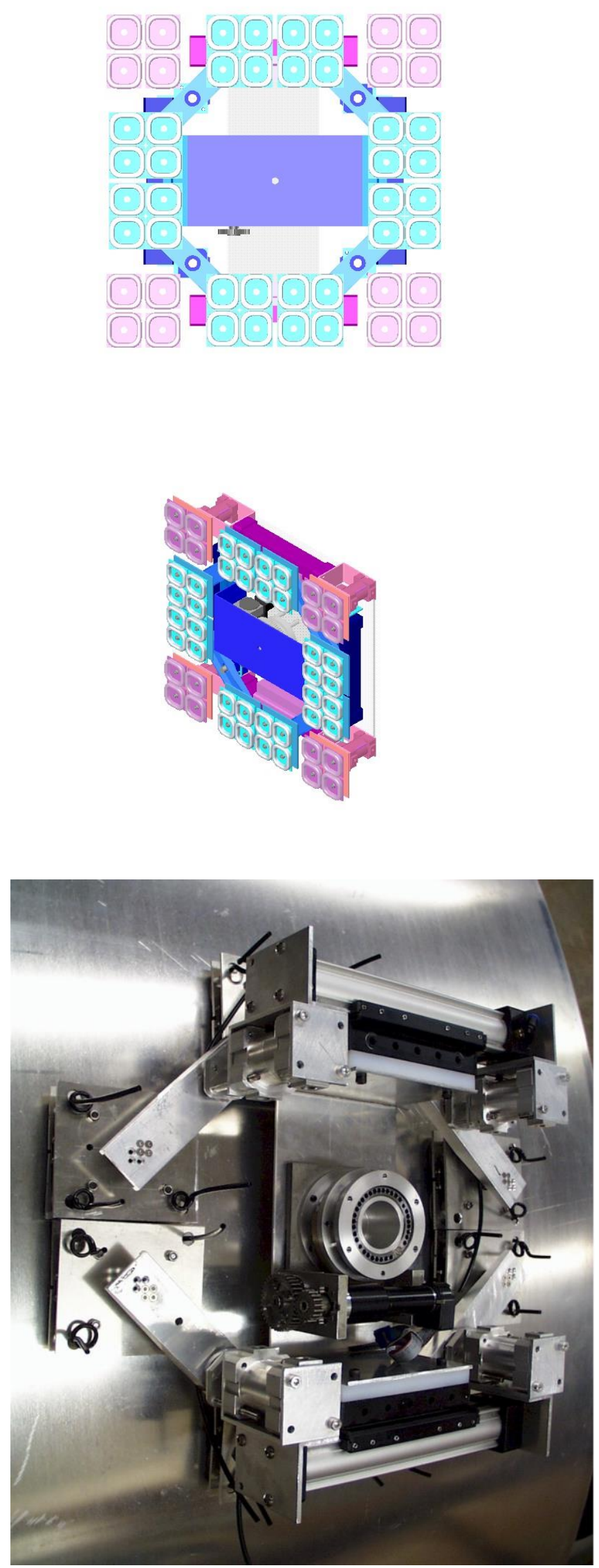